\title{
The 5D Standing Wave Braneworld with Real Scalar Field
}

\author{
Merab Gogberashvili ${ }^{1,2}$ and Pavle Midodashvili ${ }^{3}$ \\ ${ }^{1}$ Andronikashvili Institute of Physics, 6 Tamarashvili Street, 0177 Tbilisi, Georgia \\ ${ }^{2}$ Javakhishvili State University, 3 Chavchavadze Avenue, 0128 Tbilisi, Georgia \\ ${ }^{3}$ Ilia State University, 3/5 Cholokashvili Avenue, 0162 Tbilisi, Georgia
}

Correspondence should be addressed to Merab Gogberashvili; gogber@gmail.com

Received 22 October 2013; Accepted 1 December 2013

Academic Editor: Douglas Singleton

Copyright (C) 2013 M. Gogberashvili and P. Midodashvili. This is an open access article distributed under the Creative Commons Attribution License, which permits unrestricted use, distribution, and reproduction in any medium, provided the original work is properly cited.

\begin{abstract}
We introduce the new 5D braneworld with the real scalar field in the bulk. The model represents the brane which bounds collective oscillations of gravitational and scalar field standing waves. These waves are out of phase; that is, the energy of oscillations passes back and forth between the scalar and gravitational waves. When the amplitude of the standing waves is small, the brane width and the size of the horizon in extra space are of a same order of magnitude, and matter fields are localized in extra dimension due to the presence of the horizon. When oscillations are large, trapping of matter fields on the brane is provided mainly by the pressure of bulk waves. It is shown that in this case the mass of the lightest KK mode is determined by the smaller energy scale corresponding to the horizon size; that is, these modes can be created in accelerators at relatively low energies, which gives a chance to check the present model.
\end{abstract}

\section{Introduction}

Braneworld models involving large extra dimensions [1-6] have been very useful in addressing several open questions in high energy physics (e.g., the hierarchy problem and the nature of flavor). Most of the braneworlds are realized as time-independent field configurations. However, mostly within the framework of cosmological studies, there were proposed models with time-dependent metrics and matter fields, as well as branes with tensions varying in time [710]. One of the 5D braneworld models with nonstationary metric coefficients was proposed by one of us in [11-14] (for the generalization to $6 \mathrm{D}$ case, see $[15,16])$. In this scenario the braneworld was generated by standing gravitational waves coupled to a phantom-like bulk scalar field, rapid oscillations of these waves provide universal gravitational trapping of zero modes of all kinds of matter fields on the brane [17-21].

In this paper we introduce the new nonstationary $5 \mathrm{D}$ braneworld generated by standing waves of the gravitational and real scalar fields, instead of the phantom-like scalar fields of [11-14]. The model also has new features: it does not require a bulk cosmological constant and the oscillation frequency of the standing waves can be small.

\section{The Model}

We consider 5D space-time without bulk cosmological constant containing a brane and a non-self-interacting real scalar field coupled to gravity

$$
S=\int d^{5} x \sqrt{g}\left(\frac{M^{3}}{2} R+\frac{1}{2} g^{M N} \partial_{M} \varphi \partial_{N} \varphi+L_{B}\right) .
$$

Here $L_{B}$ is the brane Lagrangian and $M$ is the $5 \mathrm{D}$ fundamental scale, which relates to the $5 \mathrm{D}$ Newton constant, $G=$ $1 /\left(8 \pi M^{3}\right)$. Capital Latin indexes numerate the coordinates of 5D space-time with the signature $(+,-,-,-,-)$, and we use the units where $c=\hbar=1$. Variation of the action (1) with respect to $g_{A B}$ leads to the $5 \mathrm{D}$ Einstein equations

$$
R_{A B}-\frac{1}{2} g_{A B} R=\frac{1}{M^{3}}\left(\sigma_{A B}+T_{A B}\right) .
$$

Here the source term is the combination of the energymomentum tensors of the brane, $\sigma_{A B}$, and of the bulk scalar field,

$$
T_{A B}=\partial_{A} \varphi \partial_{B} \varphi-\frac{1}{2} g_{A B} \partial^{C} \varphi \partial_{C} \varphi .
$$


Using (3), the Einstein equations (2) can be rewritten in the form

$$
R_{A B}=\frac{1}{M^{3}}\left(\sigma_{A B}-\frac{1}{3} g_{A B} \sigma+\partial_{A} \varphi \partial_{B} \varphi\right)
$$

where $\sigma=g^{A B} \sigma_{A B}$.

To solve (4), we take the metric ansatz

$$
\begin{aligned}
d s^{2}= & \frac{e^{S}}{(1-a|r|)^{2 / 3}}\left(d t^{2}-d r^{2}\right) \\
& -(1-a|r|)^{2 / 3}\left(e^{u} d x^{2}+e^{u} d y^{2}+e^{-2 u} d z^{2}\right),
\end{aligned}
$$

where $a$ is a positive constant and $S=S(|r|)$ and $u=u(t,|r|)$ are some functions.

The metric (5) is some combination of the $5 \mathrm{D}$ generalizations of the known domain wall solution [22-24] (when $S=u=0$ ) and of the colliding plane wave solutions [2527] (when $S=a=0$ ). Similar ansatz in the $4 \mathrm{D}$ case was considered by one of us in [28].

To find a solution to the system of Einstein and background real scalar field equations, let us assume that the 5D scalar field,

$$
\varphi \equiv \varphi(t,|r|)
$$

depends only on time, $t$, and on the absolute value of the extra coordinate, $|r|$. Then its equation of motion

$$
\frac{1}{\sqrt{g}} \partial_{M}\left(\sqrt{g} g^{M N} \partial_{N} \varphi\right)=0,
$$

where

$$
\sqrt{g}=(1-a|r|)^{1 / 3} e^{S}
$$

is the determinant of the background metric (5), reduces to

$$
\varphi^{\prime \prime}+\left[2 \delta(r)-\frac{a}{1-a|r|}\right] \varphi^{\prime}-\ddot{\varphi}=0,
$$

where overdots and primes denote derivatives with respect to $t$ and $|r|$, respectively.

For the ansatz (5), the Einstein equations (4) split into the system of the equations for the metric functions

$$
\begin{gathered}
-\frac{3}{2} \dot{u}^{2}+\frac{1}{2} S^{\prime \prime}-\frac{1}{2} \frac{a}{(1-a|r|)} S^{\prime}=\frac{1}{M^{3}} \dot{\varphi}^{2}, \\
-\frac{3}{2} u^{\prime} \dot{u}=\frac{1}{M^{3}} \varphi^{\prime} \dot{\varphi}, \\
(1-a|r|)\left(u^{\prime \prime}-\ddot{u}\right)-a u^{\prime}=0, \\
-\frac{3}{2} u^{\prime 2}-\frac{1}{2} S^{\prime \prime}-\frac{1}{2} \frac{a}{(1-a|r|)} S^{\prime}=\frac{1}{M^{3}} \varphi^{\prime 2}
\end{gathered}
$$

and for the brane tensions

$$
\begin{gathered}
M^{3} \delta(r)\left(\frac{2}{3} a+S^{\prime}\right)=\sigma_{t t}-\frac{1}{3} \frac{e^{S}}{(1-a|r|)^{2 / 3}} \sigma, \\
M^{3} \delta(r)\left(\frac{2}{3} a-u^{\prime}\right) e^{-S+u}=\sigma_{x x}+\frac{1}{3}(1-a|r|)^{2 / 3} e^{u} \sigma, \\
M^{3} \delta(r)\left(\frac{2}{3} a-u^{\prime}\right) e^{-S+u}=\sigma_{y y}+\frac{1}{3}(1-a|r|)^{2 / 3} e^{u} \sigma, \\
M^{3} \delta(r)\left(\frac{2}{3} a+2 u^{\prime}\right) e^{-S-2 u}=\sigma_{z z}+\frac{1}{3}(1-a|r|)^{2 / 3} e^{-2 u} \sigma, \\
M^{3} \delta(r)\left(\frac{4}{3} a-S^{\prime}\right)=\sigma_{r r}+\frac{1}{3} \frac{e^{S}}{(1-a|r|)^{2 / 3}} \sigma .
\end{gathered}
$$

The solution to (9) and (10) is

$$
\begin{gathered}
u(t,|r|)=A \sin (\omega t) J_{0}\left(\frac{\omega}{a}-\omega|r|\right), \\
\varphi(t,|r|)=\sqrt{\frac{3 M^{3}}{2}} A \cos (\omega t) J_{0}\left(\frac{\omega}{a}-\omega|r|\right), \\
S(|r|)=\frac{3 \omega^{2}(1-a|r|)^{2}}{2 a^{2}} \\
\times A^{2}\left[J_{0}^{2}\left(\frac{\omega}{a}-\omega|r|\right)+J_{1}^{2}\left(\frac{\omega}{a}-\omega|r|\right)\right. \\
\quad-\frac{a}{\omega(1-a|r|)} J_{0}\left(\frac{\omega}{a}-\omega|r|\right) \\
\left.\quad \times J_{1}\left(\frac{\omega}{a}-\omega|r|\right)\right]-B,
\end{gathered}
$$

where $A$ and $B$ are some dimensionless constants, $J_{0}$ and $J_{1}$ are Bessel functions of the first kind, and the integration constant $\omega$ corresponds to the frequency of standing waves.

Using (12) from (11), one can easily find the brane energymomentum tensor

$$
\sigma_{A}^{B}=M^{3} \delta(r) \operatorname{diag}\left[\tau_{t}^{t}, \tau_{x}^{x}, \tau_{y}^{y}, \tau_{z}^{z}, 0\right],
$$

where the brane tensions are

$$
\begin{gathered}
\tau_{t}^{t}=2 a, \\
\tau_{x}^{x}=\tau_{y}^{y}=\frac{2}{3} a+a B+A \omega \sin (\omega t) J_{1}\left(\frac{\omega}{a}\right), \\
\tau_{z}^{z}=\frac{2}{3} a+a B-2 A \omega \sin (\omega t) J_{1}\left(\frac{\omega}{a}\right) .
\end{gathered}
$$

To interpret solution (12) as describing the brane at $r=$ 0 , which bounds the scalar and gravitational bulk standing waves, one needs to assume that the oscillatory metric functions and 5D scalar field vanish at the origin; that is,

$$
\left.u\right|_{r=0}=0,\left.\quad \varphi\right|_{r=0}=0,\left.\quad S\right|_{r=0}=0 .
$$

These conditions can be achieved assuming the relation between $\omega$ and $a$ as follows:

$$
\frac{\omega}{a}=Z_{n}^{\left(J_{0}\right)}
$$


where $Z_{n}^{\left(J_{0}\right)}$ is the $n$th zero of the function $J_{0}$, and simultaneously fixing the constant $B$ as follows:

$$
B=\frac{3 \omega^{2} A^{2}}{2 a^{2}} J_{1}^{2}\left(\frac{\omega}{a}\right) .
$$

In fact, the conditions (15) lead to the quantization, (16), of the ratio of the standing wave frequency, $\omega$, to the curvature scale, $a$.

From (12), one can also see that the metric function, $u(t,|r|)$, and the scalar field, $\varphi(t,|r|)$, having similar dependence on spatial coordinates, are oscillating $\pi / 2$ out of phase in time; that is, the energy of the oscillations is passing back and forth between the gravitational and scalar field standing waves bounded by the brane.

There are three free parameters in our model: the constant $A$ (defining the amplitude of oscillations), the curvature scale $a$ (giving the size of extra space for observers on the brane), and the ratio $Z_{n}^{\left(J_{0}\right)}=\omega / a$ (the $n$th zero of Bessel function $J_{0}$ ). Below, we consider two limiting cases corresponding to the small, $A \ll 1$, and the large, $A \gg 1$, amplitudes of bulk standing waves.

\section{The Small Extra Space}

In the first limiting case, the amplitude is $A \ll 1$, and consequently the energy of the oscillations is small. Then, neglecting the terms containing the constants $A$ and $B \sim$ $A^{2}$ in the brane tensions (14), one gets the brane energymomentum tensor,

$$
\sigma_{A}^{B} \approx M^{3} \delta(r) \operatorname{diag}\left[2 a, \frac{2}{3} a, \frac{2}{3} a, \frac{2}{3} a, 0\right],
$$

which obeys the equation of state $\mathscr{E}=3 \mathscr{P}$, with $\mathscr{E}$ and $\mathscr{P}$ being the brane energy density and pressure, respectively.

Moreover, from (12), it is clear that in this case the functions $u, S$, and $\varphi$ do not play significant role and from the very beginning one can assume the following:

$$
S(|r|)=u(t,|r|)=\varphi(t,|r|)=0
$$

and consider the metric ansatz without oscillatory metric functions as follows:

$$
\begin{aligned}
d s^{2}= & \frac{1}{(1-a|r|)^{2 / 3}}\left(d t^{2}-d r^{2}\right) \\
& -(1-a|r|)^{2 / 3}\left(d x^{2}+d y^{2}+d z^{2}\right) .
\end{aligned}
$$

This metric is $5 \mathrm{D}$ generalizations of the $4 \mathrm{D}$ domain wall solution of [22-24]. Due to the presence of the absolute value of the extra coordinate, $|r|$, the Ricci tensor at $r=0$ has $\delta$-function-like singularity which corresponds to the brane tension; see (11). Solution (20) has also new features, since, in contrast to 4D domain walls [22-24], the parameter $a$ has the opposite sign. Due to this fact, the metric (20) has the horizon at $|r|=1 / a$ in the bulk. At these points $R_{t t}$ and $R_{r r}$ components of the Ricci tensor get infinite values, while all gravitational invariants, for example, Ricci scalar,

$$
\begin{aligned}
R= & e^{-S}(1-a|r|)^{2 / 3}\left[\frac{3}{2}\left(u^{\prime 2}-\dot{u}^{2}\right)+S^{\prime \prime}\right] \\
& -a\left(2 B+\frac{8}{3}\right) \delta(r),
\end{aligned}
$$

are finite there. It resembles the situation with the Schwarzschild Black Hole; however, in contrast the determinant of our metric (20) becomes zero at $|r|=1 / a$. As the result, nothing can cross the horizon of (20), and matter fields are confined inside of the 3-brane of the width $\sim 1 / a$ in the extra space. To provide experimentally acceptable localization of matter fields, the actual size of the extra dimension must be sufficiently small, $\leq 1 / M_{H}$, where $M_{H}$ denotes the Higgs scale. So in the limiting case $A \ll 1$, the curvature scale $a$ must be large, $M_{H} \leq a \leq M$, where $M$ is the $5 \mathrm{D}$ fundamental scale. As regards the brane, its width, in fact, is defined by the horizon size.

\section{The Large Extra Space}

In the second large amplitude limiting case, it is obvious that

$$
A^{2} \sim B \gg 1 \text {. }
$$

Now, assuming that curvature scale $a$ is relatively small, the width of the brane, located at the origin of the large (of the size $\sim 1 / a$ ) but finite extra space, is determined by the metric function $S(|r|)$ in (5). Indeed, for small $a \sim \omega$, the timedependent terms in the brane tensions (14) are negligible,

$$
\sigma_{A}^{B} \approx M^{3} \delta(r) \operatorname{diag}[2 a, a B, a B, a B, 0],
$$

and the brane width is of the order of $\sim 1 /(a B)$. So trapping of matter fields is caused by the pressure of the bulk oscillations and not by the existence of the horizon in the extra space.

As an illustrative example of this trapping mechanism, let us consider localization of a real massless scalar field, $\Phi\left(x^{A}\right)$, on the brane in the background metric (5). From the 5D action,

$$
S_{\Phi}=\frac{1}{2} \int d x^{5} \sqrt{g} g^{M N} \partial_{M} \Phi \partial_{N} \Phi
$$

we obtain the Klein-Gordon equation for $\Phi\left(x^{A}\right)$ as follows:

$$
\frac{1}{\sqrt{g}} \partial_{M}\left(\sqrt{g} g^{M N} \partial_{N} \Phi\right)=0 .
$$

Using (8), equation (25) can be written as

$$
\begin{aligned}
& {\left[\partial_{t}^{2}-\frac{e^{S}}{(1-a|r|)^{4 / 3}}\left(e^{-u} \partial_{x}^{2}+e^{-u} \partial_{y}^{2}+e^{2 u} \partial_{z}^{2}\right)\right] \Phi} \\
& \quad=\frac{1}{(1-a|r|)} \partial_{r}\left[(1-a|r|) \partial_{r} \Phi\right] .
\end{aligned}
$$

In addition, it is easy to find that the assumption (22) leads to the following relation between the metric functions:

$$
\left|\frac{u(t,|r|)}{S(|r|)}\right| \ll 1
$$

Then, in the scalar field equation (26), we can drop the function $u(t, r)$ in the exponents and rewrite it as

$$
\begin{gathered}
{\left[\partial_{t}^{2}-\frac{e^{S}}{(1-a|r|)^{4 / 3}}\left(\partial_{x}^{2}+\partial_{y}^{2}+\partial_{z}^{2}\right)\right] \Phi} \\
=\frac{1}{(1-a|r|)} \partial_{r}\left[(1-a|r|) \partial_{r} \Phi\right] .
\end{gathered}
$$

We look for the solution in the form

$$
\Phi(t, x, y, z, r)=\phi\left(x^{\mu}\right) \rho(r),
$$


where Greek indexes numerate 4D coordinates, and the 4D factor of the scalar field wavefunction $\phi\left(x^{\mu}\right)$ obeys the equation

$$
\eta^{\nu \beta} \partial_{\gamma} \partial_{\beta} \phi\left(x^{\mu}\right)=-m^{2} \phi\left(x^{\mu}\right) .
$$

In what follows, we assume

$$
\phi\left(x^{\mu}\right)=e^{-i\left(E t-p_{x} x-p_{y} y-p_{z} z\right)} .
$$

Then extra dimension factor $\rho(|r|)$ of the scalar field wavefunction obeys the equation

$$
\begin{aligned}
\rho^{\prime \prime} & -\frac{a \operatorname{sgn}(r)}{1-a|r|} \rho^{\prime}-E^{2}\left[\frac{e^{S}}{(1-a|r|)^{4 / 3}}-1\right] \rho \\
& =-m^{2} \frac{e^{S}}{(1-a|r|)^{4 / 3}} \rho,
\end{aligned}
$$

with the boundary conditions

$$
\begin{gathered}
\left.\rho^{\prime}\right|_{|r|=0}=0, \\
\left.\rho\right|_{|r| \rightarrow 1 / a}=0 .
\end{gathered}
$$

For the scalar field zero mode wavefunction, $\rho_{0}(r)$, with the dispersion relation

$$
E^{2}=p_{x}^{2}+p_{y}^{2}+p_{z}^{2}
$$

equation (32) reduces to

$$
\rho_{0}^{\prime \prime}-\frac{a \text { sgn }}{(1-a|r|)} \rho_{0}^{\prime}-E^{2}\left[\frac{e^{S}}{(1-a|r|)^{4 / 3}}-1\right] \rho_{0}=0 .
$$

To show that (35) has the solution localized on the brane, we investigate the equation in two limiting regions: close to the brane $(|r| \rightarrow 0)$ and close to the horizon in the extra space $(|r| \rightarrow 1 / a)$.

In the first limiting region, $|r| \rightarrow 0$,

$$
\begin{gathered}
\left.u\right|_{|r| \rightarrow 0}=\sin (\omega t)\left[\sqrt{\frac{2}{3} B a}|r|+O\left(a^{2}|r|^{2}\right)\right], \\
\left.S\right|_{|r| \rightarrow 0}=-B a|r|+O\left(a^{2}|r|^{2}\right), \\
\left.\sqrt{g}\right|_{|r| \rightarrow 0}=1-\left(B+\frac{1}{3}\right) a|r|+O\left(a^{2}|r|^{2}\right),
\end{gathered}
$$

equation (35) has the following asymptotic form:

$$
\rho_{0}^{\prime \prime}-a \operatorname{sgn}(r) \rho_{0}^{\prime}+B E^{2} a|r| \rho_{0}=0 .
$$

This equation has the general solution

$$
\begin{array}{r}
\rho_{0}(r)=e^{a|r| / 2}\left[C_{1} A i\left(\frac{1}{4} \sqrt[3]{\frac{a^{4}}{B^{2} E^{4}}}-\sqrt[3]{\frac{B E^{2}}{a^{2}}} a|r|\right)\right. \\
\left.+C_{2} B i\left(\frac{1}{4} \sqrt[3]{\frac{a^{4}}{B^{2} E^{4}}}-\sqrt[3]{\frac{B E^{2}}{a^{2}}} a|r|\right)\right],
\end{array}
$$

where $C_{1}$ and $C_{2}$ are integration constants, and $A i$ and $B i$ are Airy functions. To fulfill the conditions (33), the constants $C_{1}$ and $C_{2}$ must obey the relation

$$
\begin{aligned}
C_{2}=-\left(2 \sqrt[3]{\frac{B E^{2}}{a^{2}}} A i^{\prime}\left(\frac{1}{4} \sqrt[3]{\frac{a^{4}}{B^{2} E^{4}}}\right)-A i\left(\frac{1}{4} \sqrt[3]{\frac{a^{4}}{B^{2} E^{4}}}\right)\right) \\
\times\left(2 \sqrt[3]{\frac{B E^{2}}{a^{2}}} B i^{\prime}\left(\frac{1}{4} \sqrt[3]{\frac{a^{4}}{B^{2} E^{4}}}\right)\right. \\
\left.-B i\left(\frac{1}{4} \sqrt[3]{\frac{a^{4}}{B^{2} E^{4}}}\right)\right)^{-1} C_{1},
\end{aligned}
$$

where $A i^{\prime}$ and $B i^{\prime}$ denote the first derivatives of Airy functions. Then, at the origin of the extra space, that is, on the brane, the function $\rho_{0}(|r|)$ will have the following series expansion:

$$
\left.\rho_{0}(r)\right|_{|r| \rightarrow 0}=C\left(1-\frac{1}{6} B E^{2} a|r|^{3}\right)+O\left(a^{4}|r|^{4}\right),
$$

where we introduced the constant

$$
\begin{gathered}
C=2 C_{1} \sqrt[3]{\frac{B E^{2}}{a^{2}}}\left(A i\left(\frac{1}{4} \sqrt[3]{\frac{a^{4}}{B^{2} E^{4}}}\right) B i^{\prime}\left(\frac{1}{4} \sqrt[3]{\frac{a^{4}}{B^{2} E^{4}}}\right)\right. \\
\left.-A i^{\prime}\left(\frac{1}{4} \sqrt[3]{\frac{a^{4}}{B^{2} E^{4}}}\right) B i\left(\frac{1}{4} \sqrt[3]{\frac{a^{4}}{B^{2} E^{4}}}\right)\right) \\
\times\left(2 \sqrt[3]{\frac{B E^{2}}{a^{2}}} B i^{\prime}\left(\frac{1}{4} \sqrt[3]{\frac{a^{4}}{B^{2} E^{4}}}\right)\right. \\
\left.-B i\left(\frac{1}{4} \sqrt[3]{\frac{a^{4}}{B^{2} E^{4}}}\right)\right)^{-1} .
\end{gathered}
$$

In the second limiting region, $|r| \rightarrow 1 / a$,

$$
\begin{gathered}
\left.u\right|_{|r| \rightarrow 1 / a}=\sin (\omega t)\left[A-\frac{1}{4} A \frac{\omega^{2}}{a^{2}}(1-a|r|)^{2}\right. \\
\left.+O\left((1-a|r|)^{3}\right)\right], \\
\left.S\right|_{|r| \rightarrow 1 / a}=-B+\frac{3}{4} A^{2} \frac{\omega^{2}}{a^{2}}(1-a|r|)^{2}+O\left((1-a|r|)^{4}\right), \\
\left.\sqrt{g}\right|_{|r| \rightarrow 1 / a}=e^{-B}(1-a|r|)^{1 / 3}+O\left((1-a|r|)^{7 / 3}\right),
\end{gathered}
$$

equation (35) has the following asymptotic form:

$$
\rho_{0}^{\prime \prime}-\frac{a \operatorname{sgn}(r)}{1-a|r|} \rho_{0}^{\prime}-\frac{c^{2}}{(1-a|r|)^{4 / 3}} \rho_{0}=0,
$$

where

$$
c^{2}=E^{2} e^{-B} .
$$


The general solution to this equation is

$$
\rho_{0}(r)=C_{3} I_{0}\left(\frac{3 c}{a} \sqrt[3]{1-a|r|}\right)+C_{4} K_{0}\left(\frac{3 c}{a} \sqrt[3]{1-a|r|}\right),
$$

where $C_{3}$ and $C_{4}$ are some integration constants and $I_{0}$ and $K_{0}$ are zero-order modified Bessel functions of the first and second kind, respectively. To fulfill the conditions (33), we must choose $C_{4}=0$ (the function $K_{0}$ is unbounded function at $|r| \rightarrow 1 / a)$. Then, for the series expansion of $\rho_{0}(r)$ at $|r| \rightarrow 1 / a$, we get

$$
\begin{aligned}
\left.\rho_{0}(r)\right|_{|r| \rightarrow 1 / a}= & C_{3}\left[1+\frac{9 c^{2}}{4 a^{2}}(1-a|r|)^{2 / 3}\right] \\
& +O\left((1-a|r|)^{4 / 3}\right) .
\end{aligned}
$$

According to (27), the action (24) of the 5D scalar filed zero mode reduces to

$$
\begin{aligned}
S_{0}=\frac{1}{2} \int d x^{4} d r[ & Q_{1}(r) \partial_{t} \phi^{2}-Q_{2}(r) \\
& \left.\times\left(\partial_{x} \phi^{2}+\partial_{y} \phi^{2}+\partial_{z} \phi^{2}\right)-Q_{3}(r) \phi^{2}\right],
\end{aligned}
$$

where integration over $r$ is performed on the finite integration region $[-1 / a,+1 / a]$, and $Q_{i}$ functions are defined as

$$
\begin{gathered}
Q_{1}(r)=(1-a|r|) \rho_{0}^{2}, \\
Q_{2}(r)=(1-a|r|)^{-1 / 3} e^{S} \rho_{0}^{2}, \\
Q_{3}(r)=(1-a|r|) \rho_{0}^{\prime 2} .
\end{gathered}
$$

It is easy to see that, for the extra dimension factor of the scalar field zero mode, $\rho_{0}(r)$, having asymptotes (40) and (46), the integral over extra coordinate $r$ in (47) is finite. This means that the scalar field zero mode wavefunction is localized on the brane. Also note that on the brane, $r=0$, due to the boundary conditions (33), the Lagrangian in the action (47) gets the standard 4D form for the massless scalar field.

To estimate the masses of KK excitations on the brane, we consider the scalar particles with zero 3 -momentum, $p^{2}=0$. Then (32) for the massive modes reduces to

$$
\rho^{\prime \prime}-\frac{a \operatorname{sgn}(r)}{1-a|r|} \rho^{\prime}+m^{2} \rho=0 .
$$

The exact general solution to this equation is

$$
\rho(r)=D_{1} J_{0}\left(\frac{m}{a}(1-a|r|)\right)+D_{2} Y_{0}\left(\frac{m}{a}(1-a|r|)\right),
$$

where $D_{1}$ and $D_{2}$ are some constants. Imposing the boundary conditions (33), we get

$$
\begin{gathered}
D_{2}=0, \\
J_{1}\left(\frac{m}{a}\right)=0,
\end{gathered}
$$

from which we get the KK mass spectrum of scalar field on the brane

$$
m_{n}=a Z_{n}^{\left(J_{1}\right)}
$$

where $Z_{n}^{\left(J_{1}\right)}$ is the $n$th zero of the Bessel function $J_{1}$. So the mass gap between the zero and the first massive modes will be

$$
\Delta_{m}=m_{1}=a Z_{1}^{\left(J_{1}\right)} \approx 3.8 a .
$$

In the limiting case of this section, the curvature scale $a$, which determines the size of extra dimension, is smaller than the scale associated with the width of the brane, $\sim a B$, where $B \gg 1$. So KK modes, having the masses $\approx 3.8 a$, can be created in accelerators at relatively low energies, what gives a chance to check this model.

\section{Conclusion}

In this paper, we have introduced the new nonstationary 5D braneworld model with the real scalar field in the bulk. The model represents single brane which bounds collective bulk oscillations of gravitational and scalar field standing waves. These waves are out of phase; that is, the energy of oscillations passes back and forth between the scalar and gravitational waves. The metric of the model has the horizon in the extra space, and, consequently, the extra space is finite for the observer on the brane. We have investigated limiting cases of large and small extra space, depending on the two dimensional parameters of the model, the curvature scale and the energy scale associated with the brane.

In the limiting case of small extra space, when the amplitude of the standing waves is small, these two parameters are of the same order of magnitude, and matter fields are localized on the brane due to the presence of the metric horizon.

In the case of large oscillations, the distance to the horizon is relatively large as compared with the brane width, and trapping of matter fields on the brane is caused mainly by the pressure of bulk oscillations. The mass of the lightest KK mode in this case is determined by the smaller energy scale associated with the horizon size, and therefore such particles can be created in future accelerators at relatively low energies.

\section{Acknowledgments}

Merab Gogberashvili was partially supported by the Grant of Shota Rustaveli National Science Foundation no. DI/8/6$100 / 12$. The research of Pavle Midodashvili was supported by Ilia State University.

\section{References}

[1] N. Arkani-Hamed, S. Dimopoulos, and G. Dvali, "The hierarchy problem and new dimensions at a millimeter," Physics Letters $B$, vol. 429, no. 3-4, pp. 263-272, 1998.

[2] I. Antoniadis, N. Arkani-Hamed, S. Dimopoulos, and G. Dvali, "New dimensions at a millimeter to a fermi and superstrings at a TeV," Physics Letters B, vol. 436, no. 3-4, pp. 257-263, 1998. 
[3] M. Gogberashvili, "Gravitational trapping for extended extra dimension," International Journal of Modern Physics D, vol. 11, no. 10, pp. 1639-1642, 2002.

[4] M. Gogberashvili, "Four dimensionality in non-compact Kaluza-Klein model," Modern Physics Letters A, vol. 14, no. 29, pp. 2025-2031, 1999.

[5] L. Randall and R. Sundrum, "Large mass hierarchy from a small extra dimension," Physical Review Letters, vol. 83, no. 17, pp. 3370-3373, 1999.

[6] L. Randall and R. Sundrum, "An alternative to compactification," Physical Review Letters, vol. 83, no. 23, pp. 4690-4693, 1999.

[7] M. Gutperle and A. Strominger, "Spacelike branes," Journal of High Energy Physics, vol. 2002, no. 4, article 18, 19 pages, 2002.

[8] M. Kruczenski, R. C. Myers, and A. W. Peet, "Supergravity Sbranes," Journal of High Energy Physics, vol. 2002, no. 5, article 39, 23 pages, 2002.

[9] V. D. Ivashchuk and D. Singleton, "Composite electric S-brane solutions with maximal number of branes," Journal of High Energy Physics, vol. 2004, no. 10, article 61, 18 pages, 2004.

[10] C. P. Burgess, F. Quevedo, R. Rabadán, G. Tasinato, and I. Zavala, "On bouncing brane worlds, S-branes and branonium cosmology," Journal of Cosmology and Astroparticle Physics, vol. 2004, no. 2, article 8, 30 pages, 2004.

[11] M. Gogberashvili and D. Singleton, "Anti-de Sitter islanduniverses from 5D standing waves," Modern Physics Letters A, vol. 25, no. 25, pp. 2131-2143, 2010.

[12] M. Gogberashvili, A. Herrera-Aguilar, and D. Malagón-Morejón, "An anisotropic standing wave braneworld and associated Sturm-Liouville problem," Classical and Quantum Gravity, vol. 29, no. 2, Article ID 025007, 14 pages, 2012.

[13] M. Gogberashvili, A. Herrera-Aguilar, D. Malagón-Morejón, R. R. Mora-Luna, and U. Nucamendi, "Thick brane isotropization in a generalized 5D anisotropic standing wave braneworld model," Physical Review D, vol. 87, no. 8, Article ID 084059, 8 pages, 2013.

[14] M. Gogberashvili, A. Herrera-Aguilar, D. Malagón-Morejón, and R. R. Mora-Luna, "Anisotropic inflation in a 5D standing wave braneworld and effective dimensional reduction," Physics Letters B, vol. 725, no. 4-5, pp. 208-211, 2013.

[15] L. J. S. Sousa, J. E. G. Silva, and C. A. S. Almeida, "A 6D standing waveBraneworld," http://arxiv.org/abs/1209.2727 .

[16] P. Midodashvili, "Localization of matter fields in the $6 \mathrm{D}$ standing wave braneworld," International Journal of Theoretical Physics, 2013.

[17] M. Gogberashvili, P. Midodashvili, and L. Midodashvili, "Localization of scalar and tensor fields in the standing wave braneworld with increasing warp factor," Physics Letters B, vol. 702, no. 4, pp. 276-280, 2011.

[18] M. Gogberashvili, P. Midodashvili, and L. Midodashvili, "Localization of gauge bosons in the 5D standing wave braneworld," Physics Letters B, vol. 707, no. 1, pp. 169-172, 2012.

[19] M. Gogberashvili, P. Midodashvili, and L. Midodashvili, "Localization problem in the $5 \mathrm{~d}$ standing wave braneworld," International Journal of Modern Physics D, vol. 21, no. 10, Article ID 1250081, 21 pages, 2012.

[20] M. Gogberashvili, "Localization of matter fields in the 5D standing wave braneworld," Journal of High Energy Physics, vol. 2012, article 56, 2012.

[21] M. Gogberashvili, O. Sakhelashvili, and G. Tukhashvili, "Numerical solutions in 5D standing wave braneworld,"
Modern Physics Letters A, vol. 28, no. 20, Article ID 1350092, 12 pages, 2013.

[22] A. H. Taub, "Isentropic hydrodynamics in plane symmetric space-times," vol. 103, pp. 454-467, 1956.

[23] A. Vilenkin, "Gravitational field of vacuum domain walls and strings," Physical Review D, vol. 23, no. 4, pp. 852-857, 1981.

[24] J. Ipser and P. Sikivie, "Gravitationally repulsive domain wall," Physical Review D, vol. 30, no. 4, pp. 712-719, 1984.

[25] U. Yurtsever, "Structure of the singularities produced by colliding plane waves," Physical Review D, vol. 38, no. 6, pp. 1706-1730, 1988.

[26] A. Feinstein and J. Ibañez, "Curvature-singularity-free solutions for colliding plane gravitational waves with broken $u$ - $v$ symmetry," Physical Review D, vol. 39, no. 2, pp. 470-473, 1989.

[27] J. B. Griffiths, Colliding Plane Waves in General Relativity, chapter 10, Oxford University Press, Oxford, UK, 1991.

[28] M. Gogberashvili, S. Myrzakul, and D. Singleton, "Standing gravitational waves from domain walls," Physical Review D, vol. 80, no. 2, Article ID 024040, 5 pages, 2009. 

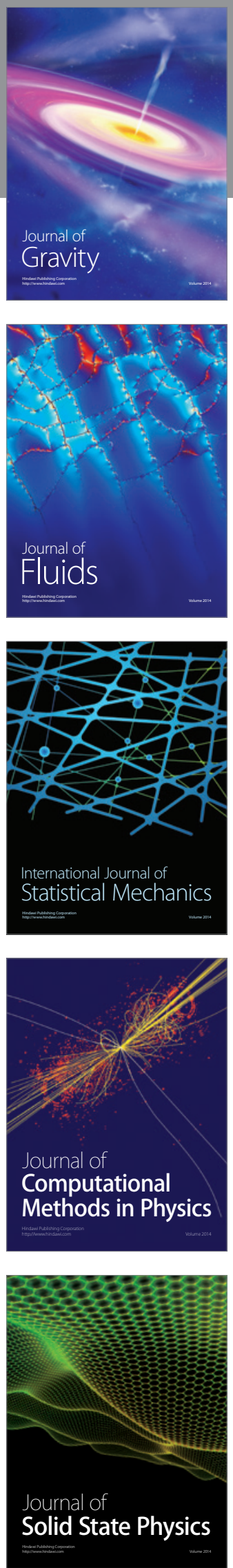

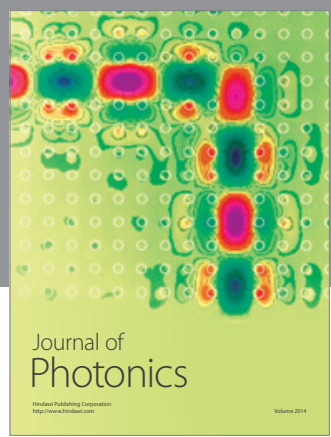

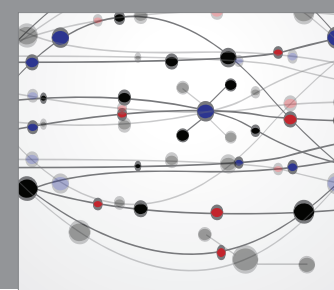

The Scientific World Journal

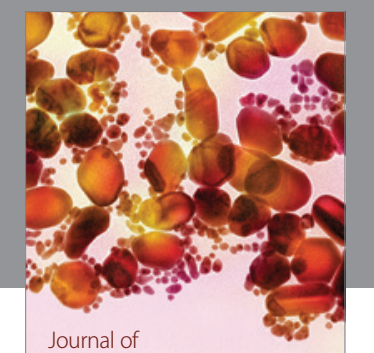

Soft Matter
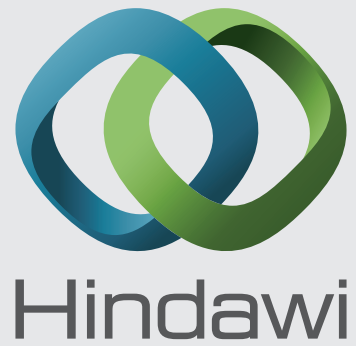

Submit your manuscripts at

http://www.hindawi.com
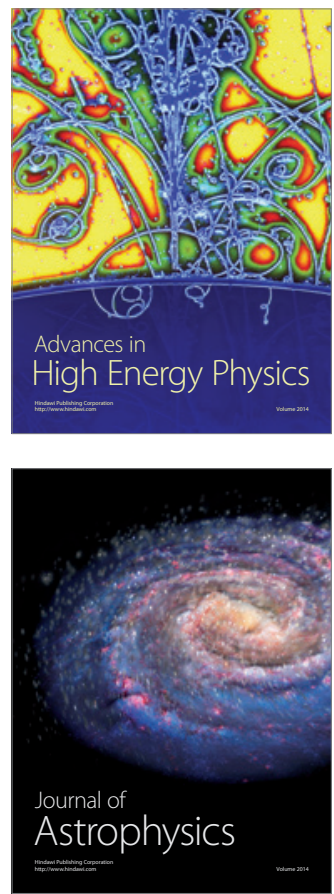
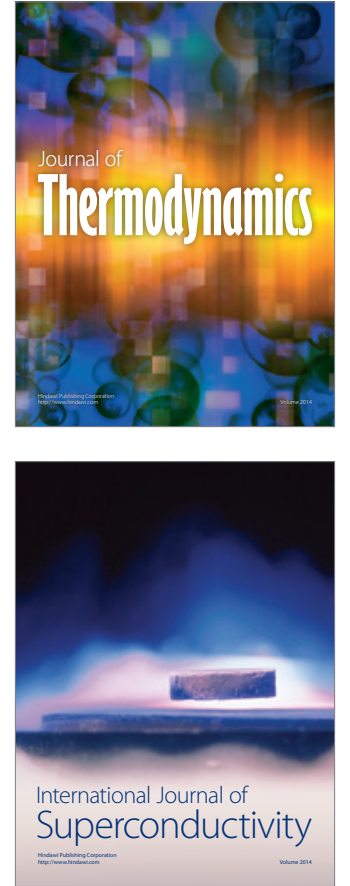
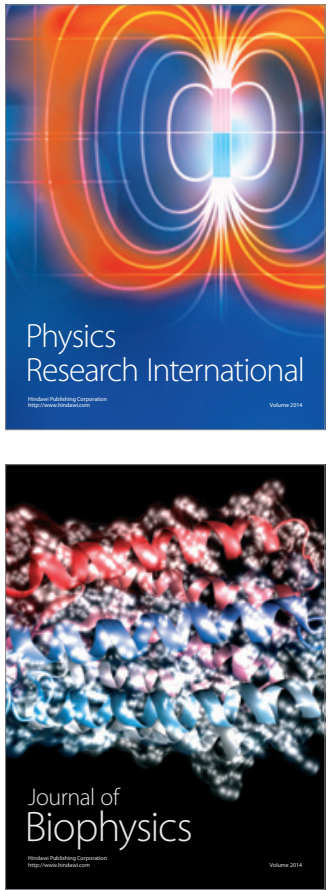
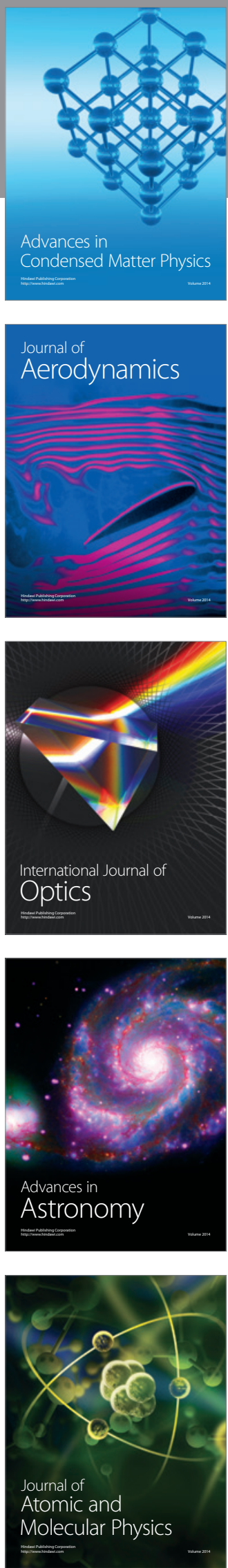